\title{
4.4. POLAR WANDERING AND CONTINENTAL DRIFT
}

\author{
S. K. RUNCORN \\ (School of Physics, The University of Newcastle upon Tyne, England)
}

\begin{abstract}
The relations between paleomagnetic observations, polar wandering curves, and changes in the speed of rotation of the Earth are discussed.

\section{RÉSUMÉ}

Les relations entre les observations paléomagnétiques, les trajectoires du pôle et leurs changements,
\end{abstract} la vitesse de la rotation de la Terre sont discutées.

1. The astronomer, studying the motion of the pole over the last hundred years, and the palaeomagnetist face the same problem: that of distinguishing between relative displacements of continental blocks and motions of the Earth (or at least the crust) as a whole with respect to the pole. Both are measuring essentially the same quantity: the variation of latitude of a place on the Earth's surface and essential to each is the assumption of a fixed axis. The astronomer is able for practical purposes to suppose that the axis of instantaneous rotation of the Earth, that is, the axis of the celestial sphere, remains fixed in space, for it is always within 0.001 of the axis of angular momentum. The precession and nutation of this axis is a separate question. The corresponding invariable axis in palaeomagnetism is that of the mean geomagnetic field, which, when averaged over times represented in a geological strata, tens or hundreds of thousands of years, is a dipole aligned along the axis of the Earth's rotation. The direction from which the astronomer measures the latitude of a place is the direction of the vertical or the vector $g$. Corresponding to this the palaeomagnetist can obtain from the remanent magnetization of rocks the mean direction of the Earth's magnetic field $(\mathbf{H})$ for some geological period. In both measurements, then, the essential question is the relation between a vector at a point on a continent and the Earth's axis of rotation.

The power of the astronomer's tool is the extreme accuracy with which he may measure directions (a hundredth of a second of arc). Because of the secular variation of the geomagnetic field, arising from magnetohydrodynamic turbulence in the Earth's fluid, electrically conducting, core, the field direction at a place at the present time diverges and over historical and geological times on the average varies by $20^{\circ}$ from that of a dipole parallel to the Earth's axis of rotation. The secular variation is

Markowitz and Guinot (eds.), Continental Drift, 80-85. () I.A.U. 
bound to be averaged out when a rock formation is sampled and the mean of the remanent magnetization vectors of the rocks taken. But the palaeomagnetist cannot indefinitely improve the accuracy of $\mathbf{H}$ by taking larger numbers of specimens, for systematic sources of error are present in his determinations. Firstly, secondary magnetization may be present in the rock as well as the primary magnetization acquired from the geomagnetic field at the time of the rock's formation. It may be possible to eliminate this secondary magnetization by "magnetic washing" techniques in the laboratory or to allow for it by examining the spatial relation of the remanent magnetization of a folded rock strata but it is impossible to do this with an accuracy of much better than a degree. Secondly, the primary magnetization, although acquired from the ambient geomagnetic field, at the time of cooling in the case of a lava or during the deposition of a sediment, may not be exactly parallel to it because of some anisotropy in the rock. Thirdly, although the original horizontal in a rock is preserved, especially in the case of a bedding plane in a sediment, the plane is never exactly specified, so that an allowance for tilting of the rocks since they became magnetized cannot be done with extreme accuracy. Thus $\mathbf{H}$ cannot be determined more accurately than, say, a degree. The geological age of the rocks in question cannot usually be known more accurately than 1 m.y. But the powerfulness of this tool at the disposal of the geophysicist is the extreme length of time over which he can recover from the rocks the field direction; even Pre-Cambrian sediments and volcanics have stable remanent moments. His measurements are almost a million times less accurate than those of the astronomers but they extend over ten million times as long a period! The variations discovered in both fields are concerned with the internal mechanics of the Earth and each should throw light on research in the other field, as I hope to show.

It is well established that for one continent for one geological time the palaeomagnetic vectors are consistent with the mean geomagnetic field being a geocentric dipole. The axis or pole of this, however, shifts with geological time. Had contemporaneous poles for different continents, determined from remanent magnetism, been in agreement for all the geological periods, polar wandering alone, in which the crust (and possibly the mantle) had moved as a whole relative to the axis of rotation, would have been a sufficient explanation of the palaeomagnetic data. The mean palaeomagnetic field - like the present one - is often specified by the angle of declination $(D)$ and the angle of dip or inclination $(I)$. At a latitude $(\lambda)$, the dipole formula gives

$$
\tan I=2 \tan \lambda .
$$

Latitudes thus determined of continents in remote geological times are compared with the evidence of climates found in the geological record and within each continent are now widely thought to be in satisfactory accord.

It is still useful, however, to represent the palaeomagnetic results from one continent by a polar wandering curve and it is found that the overall motion is a few tenths to a 
few degrees per million years. Because age determinations in geology have accuracies ranging from a tenth of a million years in the Tertiary to a million years in the Palaeozoic to tens or even hundreds of millions of years in the Pre-Cambrian, time discrimination in the geological record is poor. Thus even if the longest period of the geomagnetic secular variation was only the 500 years, which we detect from historical observations of $D$ and $I$, we could not hope palaeomagnetically to examine excursions of the pole of periods as short as a few thousand years. Palaeomagnetism therefore cannot show whether the pole moves continuously or in steps.

2. The first palaeomagnetic studies of the geologicalcolumn in Great Britain suggested polar wandering to be occurring and this led at once to the test of making palaeomagnetic measurements in another part of the world, the Grand Canyon, as a means of examining whether polar wandering alone explained the phenomenon or whether relative continental displacements had to be assumed. Similarly in the last century the variation of latitude data obtained by European observatories led to similar sets of observations being made elsewhere and the Waikiki results especially established that the main phenomenon being observed was a nutation of the pole of figure about the pole of the Earth's rotation.

Divergence between the polar wandering paths from different continents is held to have established continental drift, a hypothesis first advanced by A. Wegener by qualitative geological reasoning. There is general agreement that the continents were formerly grouped in the two continents of Gondwanaland and Laurasia at the end of the Palaeozoic and that the dispersal of the continents to form the Atlantic and Indian Oceans and to close the Tethys Sea has taken place in the last 100-200 million years. Thus, relative motions of the continental blocks of a few thousand kilometers in a few hundred million years seem established. This overall rate of movement of $1 \mathrm{~cm} /$ year is of the same order of magnitude as movements observed geodetically in the last 50 years within continents along transcurrent faults and as the spreading of the ocean floor on either side of the oceanic ridges inferred from magnetic surveys. These measurements do not show the horizontal displacements of the continents to be discontinuous although during earthquakes local displacements of meters can occur.

While relative displacements of the continents are occurring, continental drift and polar wandering cannot be separated, but it may be that prior to the dispersal of Gondwanaland and Laurasia or in the earlier evolution of the earth, or, at intervals only, polar wandering occurred and the continents remained in the same relative positions in the crust or perhaps drifted orders of magnitude less swiftly than in the last 100 million years. These are questions which will be answered as the palaeomagnetism of the Palaeozoic and Pre-Cambrian becomes better known.

It appears to be established that the pole has moved 0.220 in the last 60 years (see Markowitz, 1967). The question whether there are relative movements of the continents has not yet been resolved from the International Latitude Service data. The 
origin of this secular motion of the pole of $0.004 /$ year will now be discussed. Polar wandering inferred from palaeomagnetic observations appears to take place as well as continental drift. No explanation of drift has been suggested which is as convincing as the hypothesis that below a rigid crust, there is a spherical shell - whether this is the whole of the mantle is under dispute - in which flow, of thousands of kilometers in scale, is occurring at a rate of a few $\mathrm{cm}$ to $1 \mathrm{~m}$ per year. This is possible for the Earth's solid interior must, because of the rapid rise of temperature with depth and because of the exponential dependence of creep processes on temperature, behave, over millions of years, as a fluid below about $50 \mathrm{~km}$; although above this depth it acts as an elastic solid which fractures when stresses exceed certain limits. Such a hypothesis is also necessary to explain isostasy.

Convection currents (Runcorn, 1957) are capable of producing polar wandering. The polar wandering curves determined from Europe and North America give a mean rate since the Pre-Cambrian of $\frac{1}{3}^{\circ}$ per million years or 0 ".001 per year (Creer $e t$ al., 1957). This rate is not constant, however, and between the Devonian and the Upper Carboniferous, palaeomagnetic data from Australia (Irving, 1966) and South America (Creer, 1965) seems to show movements which may be about a factor of 5 faster.

It is not unreasonable therefore to suppose that the secular motion of the pole discovered from observations of the International Latitude Service is the same phenomena as that of palaeomagnetism. The explanation is therefore to be found in the flow pattern of the Earth's mantle. It has been argued that the recently determined low harmonics of the geopotential arise from density variations in the Earth's mantle associated with these flow patterns (Runcorn, 1966, 1967). Thus it appears that the present-day flow distribution may be worked out and as the geoid becomes better determined, it may be possible to calculate the expected secular motion of the pole. The variation of latitude data will then provide a test of the theory of convection currents in the Earth's mantle.

3. The geomagnetic secular variation has its origin in the Earth's fluid core; its time scale is $10-1000$ years, much too short to be explained by physical processes in the Earth's mantle. Hydromagnetic theory applied to the Earth's core has perhaps had its most significant success in explaining the irregular fluctuations in the length of the day, the existence of which astronomers proved from the study of the motions of the Sun, Moon, Venus, and Mercury. It is significant that the irregular fluctuations in the length of the day, the geomagnetic secular variation and the unknown process which excites the Chandlerian nutation, are the only phenomena connected with the Earth's interior which have time scales of the order of 10-100 years.

It has often been suggested that earthquakes are the source of excitation of the Chandlerian nutation, as indeed they were once suggested as a cause of the irregular fluctuations in the length of the day - the major ones occurring on a similar time scale. 
The latter is easily seen to be quantitatively wrong. Movements of mass associated with earthquakes are orders of magnitude too small to change the moment of inertia of the mantle by the few parts in $10^{7}$ required to explain the irregular changes in the length of the day. The excitation required to cause the nutation is a jolt of an axis through $0^{\prime \prime} .1$ of arc every ten or few tens of years or more frequent smaller ones. To suddenly move the axis of figure by this amount requires a change in the inertia tensor of 1 in $2 \times 10^{6}$. We can demonstrate that this is most unlikely.

Imagine the Earth to consist of a spherically symmetrical body, of moment of inertia $I$, and an external equatorial bulge of moment of inertia $I / 300$. Suppose the two can move relative to one another as may happen in the crust during an earthquake. A torque $I \omega \dot{\theta}$ applied about an equatorial axis to the sphere, will cause it to rotate about the axis at right angles to this at a rate $\dot{\theta}$, where $\omega$ is the Earth's angular velocity. The equal and opposite torque on the equatorial bulge will cause it to rotate with an angular velocity $300 \dot{\theta}$ about the same axis. Thus to move the pole by 0.1 of arc, the crust exemplified here by the equatorial bulge, $20 \mathrm{~km}$ in height, would have to move $1 \mathrm{~km}$. It is hard to imagine that this can be realistically supposed to happen in an earthquake, as Mansinha and Smylie (1967) have suggested.

We must therefore enquire if rather sudden changes of the direction of the axis of angular momentum of the Earth's mantle of the order of 0 ."1 are possible. The Earth's core is known to be rotating at a present rate of $\xi^{\circ}$ per year westwards relative to the mantle and it was pointed out by Runcorn (1954) that a change in this of $20 \%$ would imply a change in the length of the day of $1 / 300 \mathrm{sec}$ - such as was observed around 1897 - so that the total angular momentum of the Earth may remain constant. To effect this transfer of angular momentum between the core and mantle, we must postulate the existence of an impulsive torque equal to $2 \times 10^{-7}$ of the Earth's angular momentum. It is believed that this arises from the currents induced in the lower part of the Earth's mantle, which has an electrical conductivity of $0.1-10 \mathrm{ohm}^{-1} \mathrm{~cm}^{-1}$ as expected from the semi-conducting properties of silicates and oxides. The theory of this electromagnetic coupling has not yet been fully worked out but it is a necessary consequence of the observed existence of the secular variation of the geomagnetic field.

The secular variation, and the consequent mantle-core torques, arise from magnetohydrodynamic turbulence in the core. It is not simply described, having no preponderant harmonic term as has the main field. I conclude therefore that the torques exerted on the mantle are not axially symmetrical. Thus, while the component parallel to the axis of rotation changes the length of the day, that in the Earth's equatorial plane moves the axis of angular momentum away from the pole of figure. As the effects are within an order of magnitude, I conclude that this is the cause of the excitation of the Chandlerian nutation.

The astronomical data on the variation of the length of the day was once taken to imply sudden or instantaneous changes. This is physically impossible, yet one must be impressed by the short time constants involved. This seems to be possible as the time 
constants of free decay of current systems in the lower mantle are of the order of 1 year. Turbulent disturbances pass through the core as magnetohydrodynamic waves with velocities of $1-10 \mathrm{~cm}$ per second or over distances equal to the scale of the non-dipole field anomalies in months or years. This fact suggests a reason both for the comparative sharpness of the changes in the length of the day and of the jolts seen in the nutation data.

Rather sudden changes in the rate of rotation of the core have been found by the study of the westward drift of the non-dipole field since 1830. Conservation of the Earth's angular momentum also requires in the direction of the total angular momentum of the core (including that of its eddies). The geomagnetic secular variation data should allow this prediction of the above theory of the excitation of the Chandlerian wobble to be tested.

Thus, the data of the International Latitude Service is one important key in the study of the dynamic processes in the mantle and core.

\section{References}

Creer, K.M. (1965) Phil. Trans. R. Soc. Lond., A 258, 27-40.

Creer, K. M., Irving, E., Runcorn, S. K. (1957) Phil. Trans. R. Soc. Lond., A 250, 144.

Irving, E. (1966) J. geophys. Res., 71, 6025.

Mansinha, L., Smylie, D.E. (1967) J. geophys. Res., 72, 4731-4743.

Markowitz, W. (1967) the present volume, p. 25.

Runcorn, S.K. (1954) Trans. Amer. Geophys. Un., 35, 49.

Runcorn, S.K. (1957) in Gedenkboek F. A. Vening Meinesz, Mouton \& Co, The Hague, pp. 271-277.

Runcorn, S.K. (1966) Geol. Survey Canada, "The World Rift System", Paper 66-14.

Runcorn, S.K. (1967) Geophys. J. R. astr. Soc., 14, 375-384. 